\title{
Pertes de poudre entraînée par l'air sortant des installations d'atomisation à séparateur-cyclone
}

\author{
par \\ R. MARTENS et J. DE VILDER \\ Station Laitière de l'Etat, Melle \\ Centre de Recherches Agronomiques de l'Etat, Gand (Belgique)
}

\section{INTRODUCTION}

La séparation de la poudre de lait ou de sérum de l'air de séchage et de transport des tours d'atomisation va toujours de pair avec l'émission dans l'air extérieur, par la cheminée d'évacuation, d'une certaine quantité de poudre fine. L'importance de cette perte de poudre est en rapport étroit avec la valeur du produit, les normes locales relatives à la pollution de l'air et les frais et les problèmes de qualité inhérents à la récupération de cette poudre fine.

Les données pratiques sur les pertes de poudre des installations d'atomisation sont peu nombreuses. Duiven (1967) fait état de 0,3 à 1,5 p. 100 pour la poudre de lait écrémé et de 0,5 à $2,6 \mathrm{p}$. 100 pour la poudre de sérum. Hafenmayer (1972) signale des concentrations allant de 250 à $400 \mathrm{mg}$ de poudre par $\mathrm{m}^{3}$ d'air. D'après cet auteur ces pertes peuvent atteindre $800 \mathrm{mg} / \mathrm{m}^{3}$ dans des conditions d'exploitation défavorables.

Comme il n'existe aucune donnée de ce genre concernant notre pays, les recherches décrites ci-après ont été entreprises afin de procurer, par une méthode de mesurages pratiques, une idée des pertes de poudre qui se produisent dans les installations de pulvérisation industrielles, ainsi qu'une idée de l'influence de quelques importantes variables technologiques.

\section{MATERIEL ET METHODES}

\subsection{Méthode de mesure}

\subsubsection{Principe de mesure}

Pour déterminer la quantité de poudre entraînée à l'extérieur, une partie du flot d'air circonscrit par la bouche d'aspiration, a 
été aspirée pendant un certain laps de temps et la poudre entraînée a été séparée par un petit cyclone, puis pesée. Le dispositif de mesurage, tel qu'il a été décrit par Hawksley et al. (1971) est représenté schématiquement dans la figure 1.

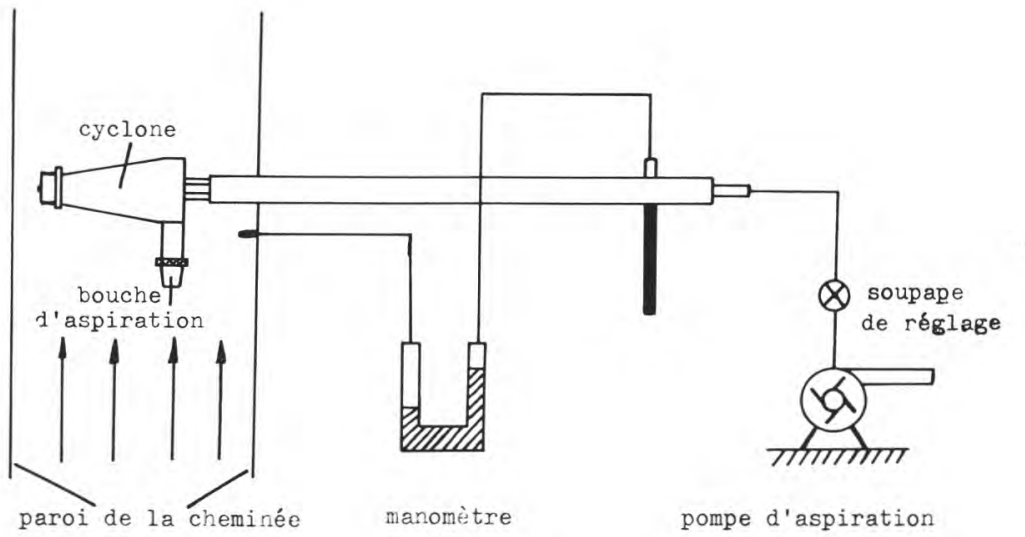

fig. 1

Dispositif de prélèvement et de mesurage des pertes de poudre.

Ce mode d'échantillonnage de l'air implique que les particules de poudre récupérées sont représentatives des particules en mouvement à l'endroit du prélèvement. Cette condition est remplie si le flot partiel est aspiré de manière isocinétique, c'est-à-dire si la vitesse d'entrée de l'air dans la bouche d'aspiration est égale à la vitesse locale du flot d'air. Dans ce cas, le poids de la poudre récupérée est égal au poids de la poudre passant par une section transversale égale à celle de la bouche d'aspiration. Le résultat d'un mesurage est exprimé en $\mathrm{mg}$ de poudre récupérée par $\mathrm{m}^{3}$ d'air aspiré.

\subsubsection{Détermination de la vitesse de passage de l'air et du débit d'air}

Pour satisfaire au principe de l'aspiration isocinétique et pour permettre le calcul du flot d'air total, les vitesses de passage de l'air doivent être déterminées dans la cheminée d'évacuation. Cette détermination a été faite par mesurage des différences de pression dans un tube de Pitot. La pression résultant de la vitesse s'y obtient 
en calculant la différence entre la pression totale $(\mathrm{Pt})$ et la pression statique (Pst) ou :

$$
\Delta \mathrm{P}=\varphi \frac{\mathrm{v}^{2}}{2 \mathrm{~g}}, \text { c.-̀̀-d. }: \mathrm{v}=\left|\frac{2 \mathrm{~g} \Delta \mathrm{P}}{\varphi}\right| 1 / 2
$$

où $\mathrm{v}=$ la vitesse de passage de l'air, en $\mathrm{m} / \mathrm{s}$,

$\mathrm{g}=$ l'accélération due à la pesanteur, c'est-à-dire $9,81 \mathrm{~m} / \mathrm{s}^{2}$,

$\Delta \mathrm{P}=$ la vitesse résultant de la pression, en $\mathrm{kPa}$,

$\varphi=$ la masse spécifique de l'air, en $\mathrm{kg} / \mathrm{m}^{3}$.

Le dispositif de mesure est reproduit schématiquement dans la figure 2 .

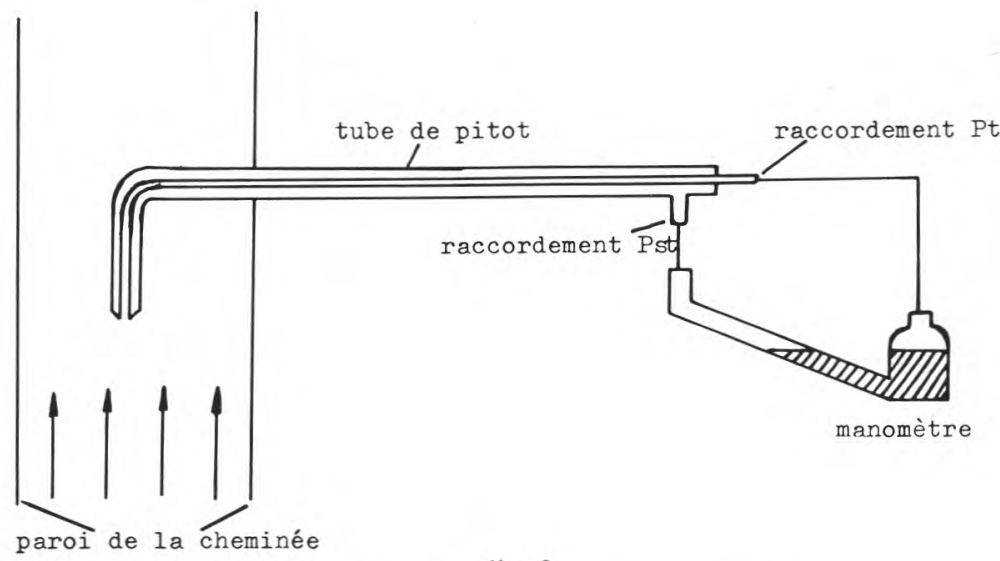

fig. 2

Dispositif de mesurage avec tube de Pitot.

Pour calculer le flot d'air total il a été fait usage d'une vitesse moyenne valable pour dix points situés sur une ligne médiane de la cheminée d'évacuation. Ces points étaient espacés de façon à se trouver juste au milieu de cinq couronnes concentriques imaginaires, de superficies identiques, comme indiqué dans la figure 3 .

Le flot d'air total passant par des tuyaux cylindriques se calcule par la formule :

$$
\mathrm{Q}\left(\mathrm{m}^{3} \mathrm{~h}\right)=\overline{\mathrm{v}} \times \frac{\pi}{4} \mathrm{~d}^{2} .3600
$$

dont on peut déduire la quantité totale de poudre perdue. 


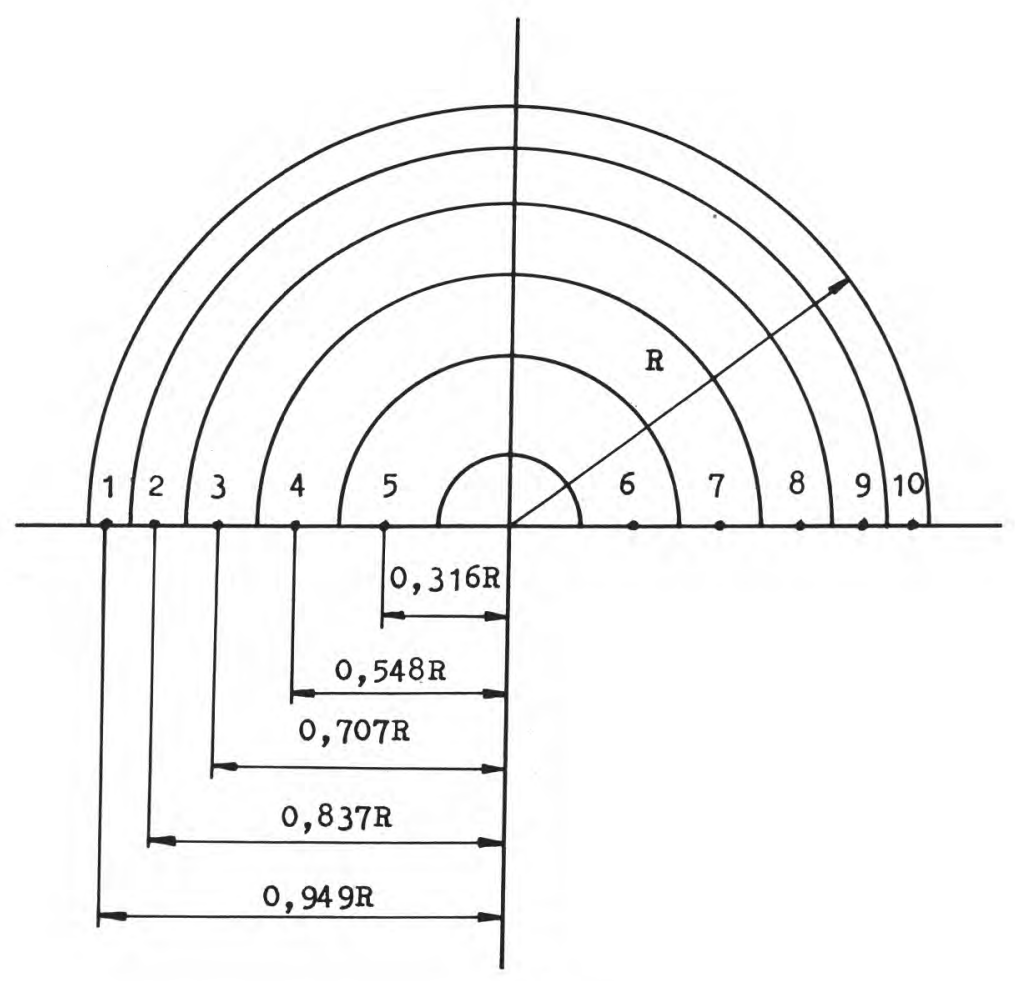

fig. 3

Points de détermination de la vitesse de passage moyenne de l'air.

\subsection{Installations d'atomisation}

Les recherches ont été effectuées sur sept installations d'atomisation industrielles, désignées ici par les lettres A à G (fig. 4), de différentes constructions et capacités, et sur une huitième, désignée par B', qui était une construction B modifiée. Certaines installations ne traitaient qu'un seul produit, soit du lait écrémé, soit du sérum ; d'autres traitaient alternativement ces deux produits. La majorité des installations ont été étudiées à plusieurs reprises, à des intervalles plus ou moins rapprochés.

Dans la cheminée d'évacuation, l'endroit choisi pour les points de mesurage était situé dans une section rectiligne, aussi éloignée que possible du ou des ventilateurs d'aspiration. Les situations des 


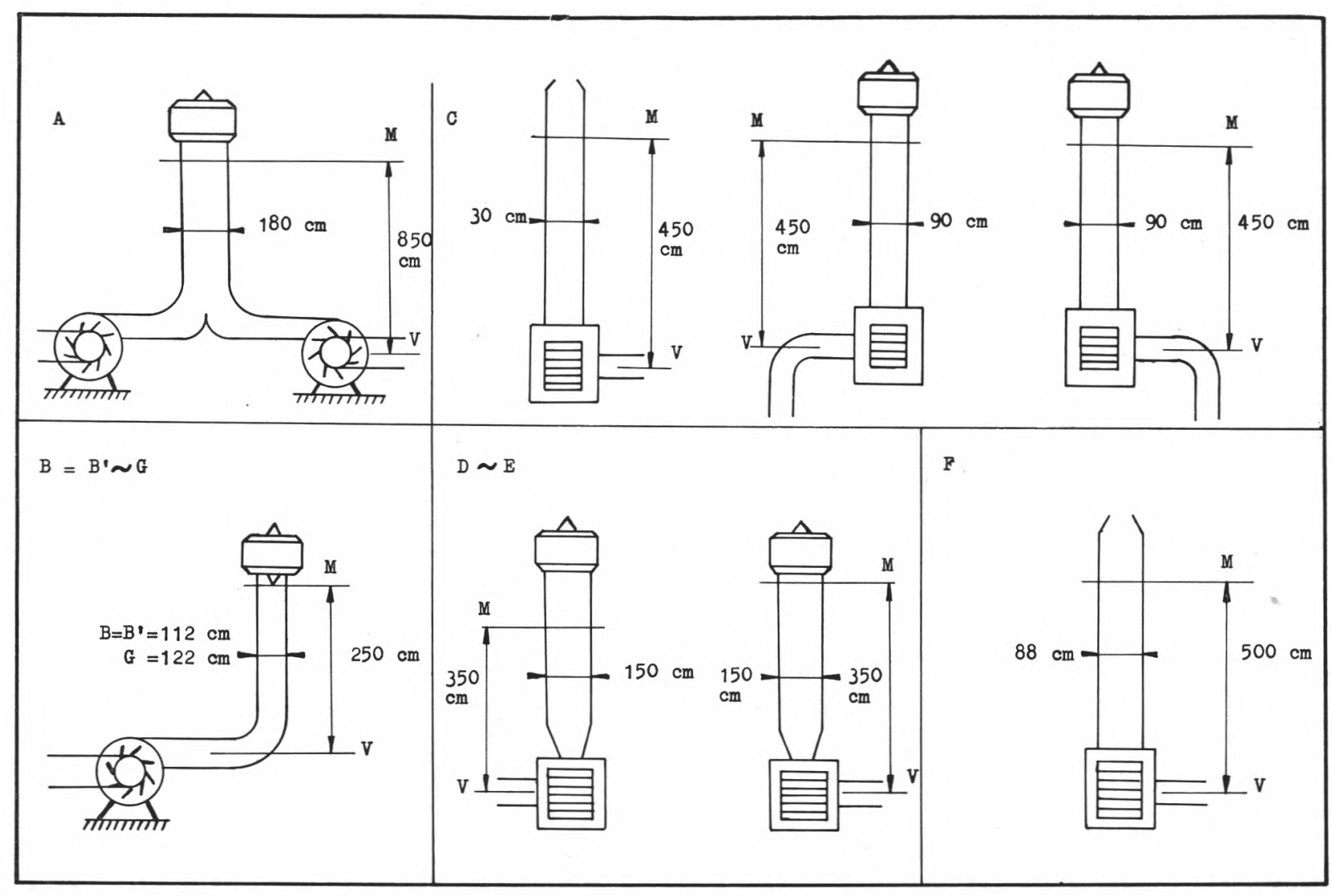


lieux de mesurage $(\mathrm{M})$ par rapport à ce ou ces ventilateurs sont indiquées schématiquement dans la figure 4. Seule l'installation C était pourvue d'une cheminée séparée pour l'évacuation de l'air du cyclone de soutirage.

\subsection{Déterminations et analyses}

Au cours des différents essais, nous avons enregistré à intervalles réguliers, en plus des résultats des mesurages de la perte de poudre, des données technologiques telles que la température de l'air de séchage et celle du concentré, ainsi que la production de poudre.

La teneur en eau des échantillons de concentré et de poudre a été déterminée par dessiccation à $102^{\circ} \mathrm{C}$ jusqu'à obtention d'un poids constant, selon la norme FIL/IDF 26 (1964). Le poids d'emballage des échantillons de poudre a été calculé d'après le volume de $50 \mathrm{~g}$ après 600 coups du voluménomètre à secousses JEL ST 2. La composition granulométrique des poudres a été déterminée par tamisage et examen microscopique. La méthode décrite par Buma (1971) a été suivie pour déterminer la densité des particules et le volume des vacuoles.

\section{RESULTATS}

\subsection{Fiabilité de la méthode de mesure}

La quantité de poudre perdue par la cheminée est calculée d'après la concentration de la poudre dans le flot aspiré. Cette façon de procéder suppose que la quantité de poudre récupérée est représentative pour la teneur en poudre du flot d'air total. Cette hypothèse n'est toutefois valable que si le flot de poudre est uniformément réparti sur toute la section transversale de la cheminée ce qui n'est pas toujours le cas dans la pratique. On ne sait pas d'avance si la poudre fine présente dans les cheminées d'évacuation des installations d'atomisation à séparateur-cyclone est répartie uniformément ou non sur les sections transversales. C'est pourquoi il était nécessaire de procéder à des mesurages en différents points. Ces mesurages ont fourni un premier groupe de résultats.

Le nombre de points de mesure indispensables dépend évidemment du degré d'uniformité de la répartition de la poudre. Il n'est cependant pas le seul facteur qui ait une influence sur la fiabilité : la durée des mesurages a aussi son importance. En effet, le flot de poudre n'est jamais constant et la quantité de poudre récupérée est une moyenne pour la durée de mesurage choisie. Dans nos recherches, chaque mesurage a duré $30 \mathrm{mn}$, une durée plus courte 
ne permettant pas d'éliminer suffisamment l'influence de variations accidentelles.

\subsubsection{Choix des points de mesure}

Le profil des vitesses de passage de l'air dans une-cheminée d'évacuation donne une idée de la nature des courants locaux et de la distribution de la poudre dans la section transversale de la cheminée. Les profils de ce genre sont influencés par les dimensions de la cheminée, par la situation du lieu de mesure par rapport au(x) ventilateur(s) d'aspiration et par les coudes et obstacles en amont ou en aval du lieu de mesure.

La figure 5 reproduit trois types différents de profils de vitesses. Nous y avons dessiné deux profils, a et $b$, relatifs à deux diamètres
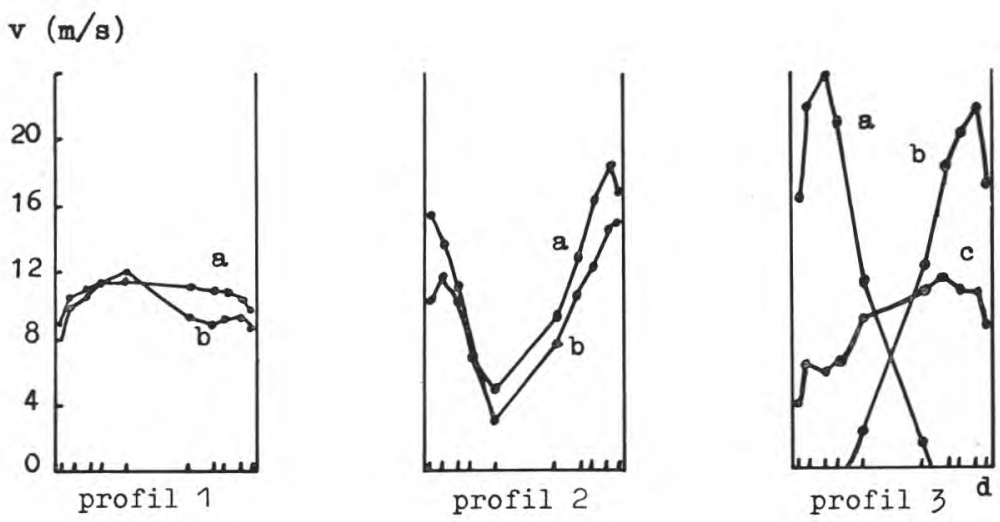

fig. 5

Profils de vitesses de passage de l'air.

situés perpendiculairement dans un même plan. Dans le graphique 3, nous avons dessiné en outre le profil c, relatif à des points situés sur un diamètre qui forme un angle de $45^{\circ} \mathrm{C}$ par rapport aux diamètres $a$ et $b$.

Le profil 1 (tours $\mathrm{A}, \mathrm{C}$ et $\mathrm{F}$ ) était caractérisé par une courbe de vitesse uniforme, d'allure égale. La distance entre le ventilateur d'aspiration et le lieu de mesure était d'au moins $450 \mathrm{~cm}$, sans coude ni autre obstacle. La direction d'écoulement de l'air était pratiquement parallèle à l'axe de la cheminée et le choix des points de mesure était peu limité. 

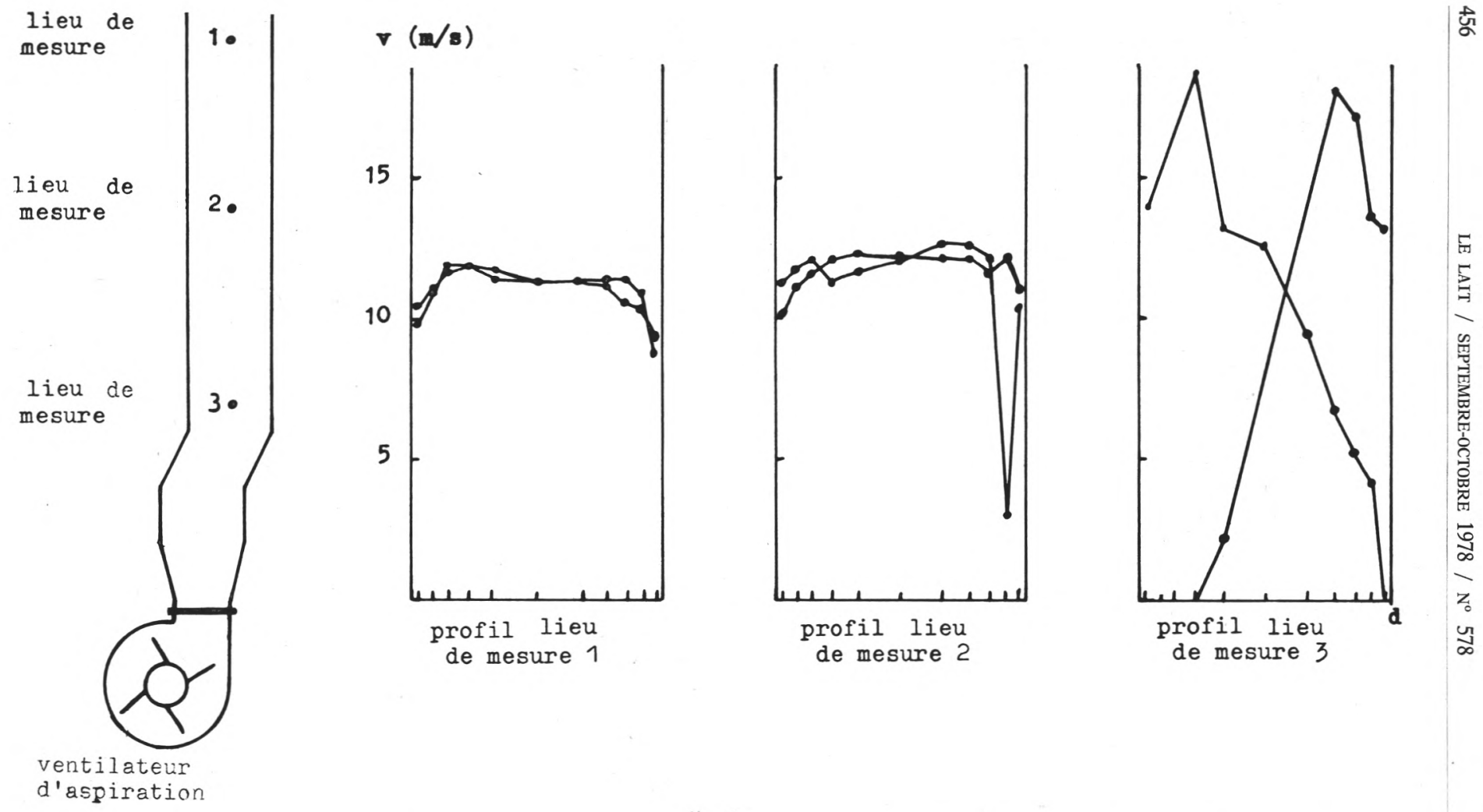

fig. 6

Profils de vitesses de passage en différents lieux de mesure. 
Dans le cas du profil 2 (tours B, G et B'), par contre, où la distance entre le ventilateur d'aspiration et le lieu de mesure n'était que de $250 \mathrm{~cm}$, la cheminée présentait un coude de $90^{\circ}$ et un cône était fixé à hauteur du lieu de mesure. Ce dernier provoquait un ralentissement considérable du courant central et une accélération des courants le long de la paroi. Dans ce cas, le choix des points de mesure restait limité à ceux où la vitesse était la plus proche de la vitesse moyenne.

Dans le profil 3 (tours D et $\mathrm{E}$ ) on relevait des vitesses très variées, tout selon l'endroit de la section transversale, allant de points à très faible vitesse à des points où la vitesse de passage dépassait $20 \mathrm{~m} / \mathrm{s}$. Il ne s'agissait plus d'écoulements parallèles à l'axe du tuyau, mais d'un déplacement d'air ondulatoire et le choix des points de mesure était très limité. Ce mouvement ondulatoire de l'air sortant doit être attribué à la distance trop courte entre le lieu de mesure et le ventilateur d'aspiration, comme le montre la figure 6. Celle-ci reproduit les profils de vitesses tels qu'ils ont été mesurés à différentes distances du ventilateur d'aspiration. Il en ressort clairement que l'endroit de mesure détermine le type de profil de vitesses et, par conséquent, le choix des différents points de mesure.

Quoique le choix d'un point de mesure situé sur une partie plane du profil de vitesses donne moins lieu à des mesurages erronés, on ne peut néanmoins pas écarter sans plus un point situé sur une partie non plane du profil si la distribution des vitesses reste constante dans le temps. De nombreuses mesures de vitesses de passage, effectuées à hauteur du même lieu de mesure, ont démontré que la distribution des vitesses ne variait que peu dans une installation donnée ou pour un produit donné, ceci aussi bien dans le courant d'une journée de production donnée que d'une journée de production à l'autre.

\subsubsection{Quantité moyenne de poudre perdue}

Du fait des variations possibles de la teneur en poudre de l'air sortant, en fonction du lieu et (ou) du temps, un chiffre fiable ne peut être obtenu qu'après des mesurages répétés. Ceux-ci peuvent être répétés en un même point ou en différents points de la section transversale.

Le tableau 1 indique, pour quelques installations, les pertes de poudre, exprimées en $\mathrm{mg} / \mathrm{m}^{3}$, relevées par des mesurages répétés en un même point. Ces résultats montrent que dans certains cas et pendant une période de fonctionnement de $2 \mathrm{~h}$ à $2 \mathrm{~h} 30 \mathrm{mn}$ (quatre ou cinq mesurages) la perte est restée remarquablement constante, même lorsque le profil des vitesses n'était pas régulier (tests 18 et 23 , type 3), mais que dans d'autres cas elle se modifiait brusquement et considérablement (tests 13 et 15, type 1) en dépit d'un profil 
TABLEAU 1

Pertes de poudre au cours de mesurages répétés au même point d'une section transversale de la cheminée

\begin{tabular}{|c|c|c|c|c|c|}
\hline \multirow{3}{*}{$\begin{array}{c}\text { Répétition } \\
n^{\circ}\end{array}$} & \multicolumn{5}{|c|}{ Pertes de poudre, en $\mathrm{mg} / \mathrm{m}^{3}$} \\
\hline & \multicolumn{2}{|c|}{$\begin{array}{l}\text { Tour A } \\
\text { poudre de lait } \\
\text { écrémé }\end{array}$} & \multirow{2}{*}{$\begin{array}{c}\begin{array}{c}\text { Tour C } \\
\text { poudre de } \\
\text { sérum }\end{array} \\
\text { test } 15\end{array}$} & \multirow{2}{*}{$\begin{array}{c}\begin{array}{c}\text { Tour D } \\
\text { poudre de } \\
\text { lait écrémé }\end{array} \\
\text { test } 18\end{array}$} & \multirow{2}{*}{$\begin{array}{c}\begin{array}{c}\text { Tour } \mathrm{E} \\
\text { poudre de } \\
\text { lait écrémé }\end{array} \\
\text { test } 23\end{array}$} \\
\hline & test 8 & test 13 & & & \\
\hline 1 & 179 & 95 & 543 & 120 & 184 \\
\hline 2 & 160 & 79 & 647 & 110 & 193 \\
\hline 3 & 196 & 89 & 658 & 126 & 184 \\
\hline 4 & 178 & 129 & 589 & 116 & 186 \\
\hline 5 & 194 & 143 & 599 & & 182 \\
\hline perte moyenne & 181 & 107 & 607 & 118 & 186 \\
\hline p. 100 & 0,63 & 0,39 & 3,5 & 0,47 & 0,84 \\
\hline
\end{tabular}

bien étalé. Ceci indique, d'une part, une bonne reproductibilité de la méthode de mesure et, d'autre part, une certaine influence de facteurs technologiques. Cette dernière influence est mise bien en évidence par une comparaison des résultats des tests 8 et 13 de la tour A. Après $1 \mathrm{~h} 30 \mathrm{mn}$ de fonctionnement, ou trois répétitions de $30 \mathrm{mn}$ chacune, le test 13 a révélé une augmentation soudaine et importante de la perte de poudre, conséquence du passage à un concentré moins riche en extrait sec. Ceci ne s'est pas produit dans le test 8. Dans le test 15 , de la tour $\mathrm{C}$, une diminution de 46,8 à 41,6 p. 100 de la teneur en extrait sec du concentré est allée de pair avec une augmentation de 543 à $658 \mathrm{mg} / \mathrm{m}^{3}$ de la perte de poudre et une diminution, d'ailleurs escomptée, de 3,80 à 3,48 p. 100 de la teneur en eau. Une augmentation ultérieure, de 41,6 à 43,8 p. 100, de la teneur en extrait sec du concentré, a résulté de nouveau en une diminution de la perte de poudre, de 658 à $589 \mathrm{mg} / \mathrm{m}^{3}$, et en une augmentation de la teneur en eau, de 3,48 à 3,74 p. 100 . 
Le tableau 2 indique, pour quelques installations, les pertes de poudre relevées par des mesurages répétés en différents points de la section transversale de la cheminée (cf. fig. 3). Ces résultats montrent clairement que la perte de poudre est pratiquement constante, parfois sur toute l'étendue du profil (test 7 de la tour A

TABLEAU 2

Pertes de poudre au cours de mesurages répétés en différents points de la section transversale de la cheminée

\begin{tabular}{|c|c|c|c|c|}
\hline \multirow{3}{*}{$\begin{array}{c}\text { Point de } \\
\text { mesure } \\
n^{\circ}\end{array}$} & \multicolumn{4}{|c|}{ Perte de poudre, en $\mathrm{mg} / \mathrm{m}^{3}$} \\
\hline & \multicolumn{2}{|c|}{$\begin{array}{l}\text { Tour A } \\
\text { poudre de lait } \\
\text { écrémé }\end{array}$} & \multirow{2}{*}{$\begin{array}{c}\begin{array}{c}\text { Tour C } \\
\text { poudre de } \\
\text { sérum }\end{array} \\
\text { test } 17\end{array}$} & \multirow{2}{*}{$\begin{array}{c}\begin{array}{c}\text { Tour } \mathrm{F} \\
\text { poudre de } \\
\text { lait écrémé }\end{array} \\
\text { test } 28\end{array}$} \\
\hline & test 6 & test 7 & & \\
\hline 3 & 124 & 173 & 606 & \\
\hline 4 & 123 & 182 & 610 & 166 \\
\hline 5 & 125 & 198 & 631 & 175 \\
\hline $\mathrm{C}$ & & & 622 & 188 \\
\hline 6 & 77 & 199 & 625 & 194 \\
\hline 7 & 86 & 199 & 639 & 206 \\
\hline 8 & 93 & 208 & & \\
\hline \multirow{3}{*}{$\begin{array}{c}\text { perte moyenne } \\
\text { p. } 100\end{array}$} & \multirow{3}{*}{$\begin{array}{r}105 \\
0,32\end{array}$} & \multirow{3}{*}{$\begin{array}{r}193 \\
0,67\end{array}$} & \multirow{3}{*}{$\begin{array}{r}622 \\
3,6\end{array}$} & \\
\hline & & & & \\
\hline & & & & 1,1 \\
\hline
\end{tabular}

et test 17 de la tour $\mathrm{C}$ ), ce qui indique une répartition uniforme de la poudre dans le flot d'air. Certaines différences systématiques, comme dans le test 6 de la tour A, sont donc imputables à des 
variations en fonction du temps, telles qu'une modification de la teneur en extrait sec du concentré, et non en fonction du lieu.

Il ressort de ce qui précède que les mesurages répétés en différents points n'ont révélé que peu de différences en perte de poudre. C'est pourquoi une méthode simplifiée a été suivie pour de nombreuses déterminations de routine, qui visaient uniquement à procurer un chiffre moyen quant à la quantité de poudre perdue. Cette méthode consistait à n'effectuer qu'un ou tout au plus deux mesurages en deux points nettement distincts, la durée totale des mesurages étant de $1 \mathrm{~h}$ ou $2 \mathrm{~h}$. Les résultats de quelques-uns de ces tests figurent dans le tableau 3. Ils montrent une fois de plus que les chiffres obtenus en des points nettement distincts de la section transversale concordent généralement très bien et fournissent par conséquent des valeurs moyennes suffisamment fiables.

TABLEAU 3

Pertes de poudre des installations $\mathrm{B}$ et $\mathrm{C}$, d'après des mesurages en deux points

\begin{tabular}{|c|c|c|c|c|}
\hline & \multicolumn{2}{|c|}{ Tour B } & \multicolumn{2}{|c|}{ Tour C } \\
\hline & $\begin{array}{l}\text { poudre de } \\
\text { sérum } \\
\text { test } 12\end{array}$ & $\begin{array}{l}\text { poudre de } \\
\text { sérum } \\
\text { test } 26\end{array}$ & $\begin{array}{l}\text { poudre de } \\
\text { lait écrémé } \\
\text { test } 14\end{array}$ & $\begin{array}{l}\text { poudre de } \\
\text { lait écrémé } \\
\text { test } 21\end{array}$ \\
\hline Vitesse de l'air $(\mathrm{m} / \mathrm{s})$ : & & & & \\
\hline - premier point $\left(\mathrm{n}^{\circ}\right)$ & $9,4(3)$ & $8,1(4)$ & $15,4(3)$ & $14,1(3)$ \\
\hline - deuxième point $\left(\mathrm{n}^{\circ}\right)$ & $12,6(7)$ & $13,0(7)$ & $15,1(6)$ & $14,5(8)$ \\
\hline Perte de poudre $\left(\mathrm{mg} / \mathrm{m}^{3}\right)$ & & & & \\
\hline - premier point & $\begin{array}{l}314 \\
305\end{array}$ & $\begin{array}{l}187 \\
186\end{array}$ & 552 & 365 \\
\hline — deuxième point & $\begin{array}{l}310 \\
309\end{array}$ & $\begin{array}{l}196 \\
192\end{array}$ & 576 & 365 \\
\hline Moyenne : $\mathrm{mg} / \mathrm{m}^{3}$ & 307 & 191 & 564 & 365 \\
\hline p. 100 & 1,58 & 1,00 & 3,30 & 1,35 \\
\hline
\end{tabular}




\subsection{Influence de variables technologiques}

Le tableau 4 présente les résultats de tests répétés, selon la méthode simplifiée, effectués à différents jours dans les installations $A, B, D$ et $E$ au cours du séchage de lait écrémé. Il met en évidence que la perte de poudre présente des variations assez considérables, non seulement d'une installation à l'autre, mais même

\section{TABLEAU 4}

Pertes de poudre des installations A, B, D et E au cours de tests effectués à différentes dates

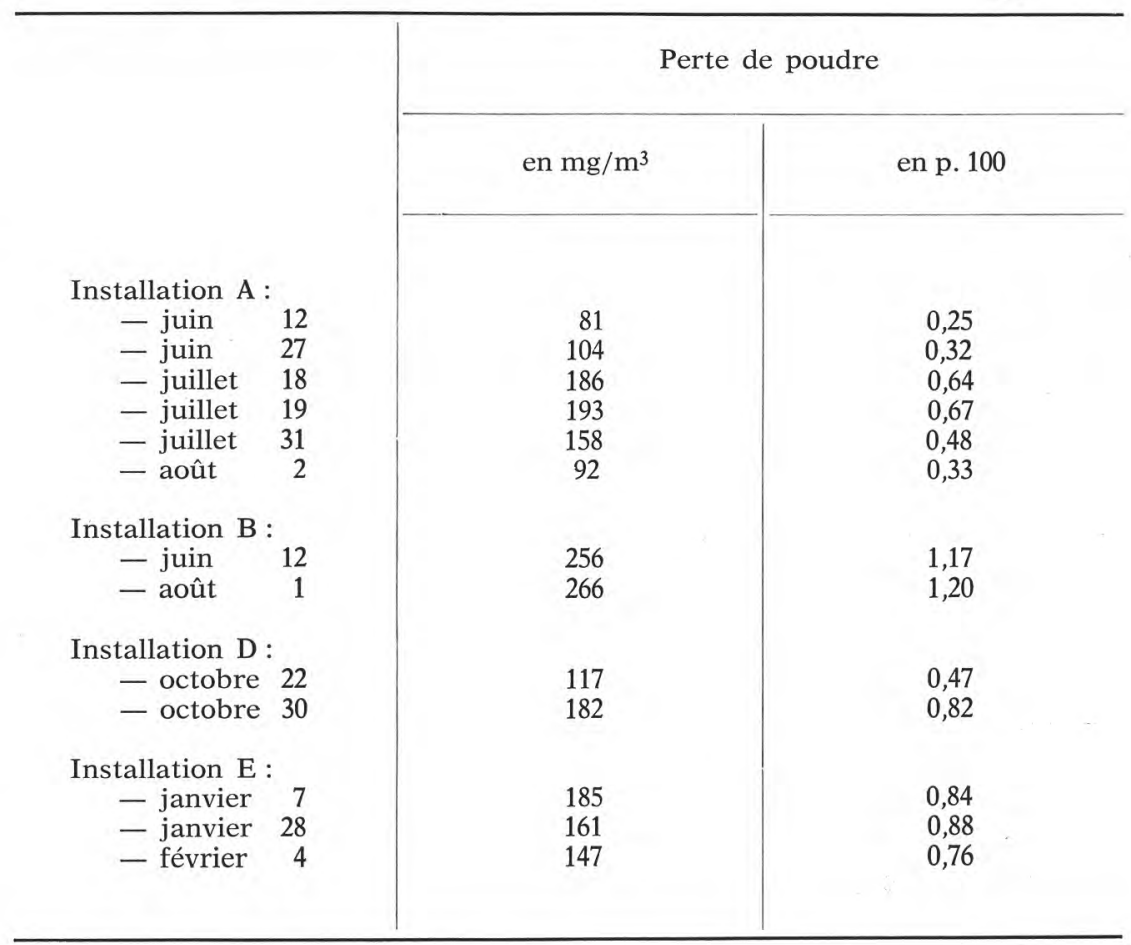

pour une installation donnée. Ceci était à prévoir, certaines conditions de fabrication, dont les propriétés physiques du concentré, influant sur la composition granulométrique et la densité de la poudre, et par conséquent sur l'effiçacité des séparateurs-cyclones. 
Nous avons étudié l'influence exercée par les variables technologiques sur la perte de poudre dans les conditions pratiques en effectuant deux mesurages consécutifs, de $30 \mathrm{mn}$ chacun, au même point de la section transversale, pour trois valeurs différentes du facteur variable.

\subsubsection{Teneur en extrait sec du concentré}

Les résultats de trois tests relatifs à l'influence exercée par la teneur en extrait sec du concentré sur la perte de poudre sont reproduits dans le tableau 5 .

\section{TABLEAU 5}

Influence de la teneur en extrait sec du concentré sur la perte de poudre

\begin{tabular}{|c|c|c|c|c|}
\hline Installation & $\begin{array}{l}\text { Produit } \\
\text { test } n^{\circ}\end{array}$ & $\begin{array}{l}\text { Extrait sec } \\
\text { du concentré }\end{array}$ & $\begin{array}{c}\text { Concentration } \\
\text { de poudre dans } \\
\text { l'air évacué } \\
\mathrm{mg} / \mathrm{m}^{3}\end{array}$ & $\begin{array}{c}\text { Perte } \\
\text { de poudre } \\
\text { p. } 100\end{array}$ \\
\hline \multirow[t]{2}{*}{ B } & $\begin{array}{l}\text { sérum } \\
\text { test } 30\end{array}$ & $\begin{array}{l}40,5 \\
41,6 \\
47,8\end{array}$ & $\begin{array}{l}486 \\
470 \\
348\end{array}$ & $\begin{array}{l}3,4 \\
2,9 \\
1,9\end{array}$ \\
\hline & $\begin{array}{l}\text { sérum } \\
\text { test } 31\end{array}$ & $\begin{array}{l}43,1 \\
48,2 \\
49,8\end{array}$ & $\begin{array}{l}403 \\
356 \\
265\end{array}$ & $\begin{array}{l}2,5 \\
2,0 \\
1,2\end{array}$ \\
\hline $\mathrm{B}^{\prime}$ & $\begin{array}{c}\text { lait } \\
\text { écrémé } \\
\text { test } 32\end{array}$ & $\begin{array}{l}40,1 \\
43,4 \\
48,0\end{array}$ & $\begin{array}{l}616 \\
458 \\
282\end{array}$ & $\begin{array}{l}3,2 \\
2,1 \\
1,2\end{array}$ \\
\hline
\end{tabular}

Les données de ce tableau montrent clairement que lorsque la teneur en extrait sec du concentré était portée de $40-43$ p. 100 à $48-50$ p. 100 , la concentration de poudre dans l'air sortant diminuait considérablement.

Du fait de l'augmentation simultanée de la production horaire, la perte de poudre, exprimée par rapport à la quantité totale de poudre produite, a été réduite dans ces conditions de parfois plus de moitié.

L'influence considérable de la teneur en extrait sec du concentré s'est manifestée de façon particulièrement frappante au cours 
du test 32, effectué dans l'installation B', où le taux de perte de 3,2 p. 100, exceptionnellement élevé pour une poudre de lait écrémé, a été réduit à 1,2 p. 100. Cette influence favorable est certainement due dans une large mesure à la plus grande viscosité du concentré à plus haute teneur en extrait $\mathrm{sec}$, qui résultait en une pulvérisation plus grossière et une poudre à grains de plus grand diamètre, à particules plus denses, à poids d'emballage plus élevé et à moindre volume de vacuoles. L'influence de l'état du concentré sur les caractéristiques physiques de la poudre a été étudiée plus en détail par de Vilder et al. (sous presse).

\subsubsection{Température de l'air entrant}

On peut se demander si l'influence favorable de plus hautes teneurs en extrait sec du concentré sur l'émission de poudre est due uniquement aux caractéristiques physiques modifiées de la poudre ou, aussi, à la plus forte concentration de poudre dans l'air de séchage, en d'autres termes à la charge du cyclone.

Dans trois tests, la concentration de poudre dans l'air de séchage a été influencée par une augmentation de la température à l'entrée, les caractéristiques du concentré restant constantes. Le tableau 6 donne les résultats d'un test effectué dans la tour B avec de la poudre de sérum.

\section{TABLEAU 6}

Influence de la température d'entrée de l'air de séchage sur les pertes de poudres

\begin{tabular}{c|c|c|c}
\hline $\begin{array}{c}\text { Température de l'air } \\
\left({ }^{\circ} \text { C) de séchage }\right.\end{array}$ & $\begin{array}{c}\text { Concentration } \\
\text { de poudre dans l'air } \\
\text { de séchage } \\
\mathrm{g} / \mathrm{m}^{3}\end{array}$ & $\begin{array}{c}\text { Concentration } \\
\text { de poudre dans l'air } \\
\text { évacué } \\
\mathrm{mg} / \mathrm{m}^{3}\end{array}$ & Perte p. 100 \\
\cline { 2 - 3 } 160 & 17,3 & 244 & 1,4 \\
168 & 22,2 & 273 & 1,2 \\
180 & 28,4 & 294 & 1,0 \\
\hline
\end{tabular}

Il ressort des chiffres de ce tableau qu'une nette augmentation de la concentration de poudre dans l'air de séchage est effectivement allée de pair avec une légère augmentation de la concentration 
de poudre dans l'air sortant, mais que la perte de poudre exprimée par $100 \mathrm{~kg}$ de produit séché a nettement diminué.

L'augmentation de la température d'entrée de l'air de séchage va de pair avec la production de poudre à particules de plus faible densité, poids d'emballage moins élevé et volume de vacuoles plus considérable. L'influence de la température de l'air sur les caractéristiques physiques mentionnées est toutefois minime, comme déjà démontré, entre autres par de Vilder et al. (1976) pour la poudre de lait entier. La charge plus élevée du cyclone est donc plus importante que l'effet défavorable des caractéristiques physiques de la poudre.

\subsubsection{Température du concentré}

Le chauffage du concentré influe favorablement sur la qualité bactériologique de la poudre et augmente le rendement de l'installation.

Pour étudier l'effet que le chauffage du concentré exerce sur l'émission de poudre, nous avons fait augmenter la température d'un concentré de lait écrémé, à 50 p. 100 de matière sèche, dans un échangeur de chaleur tubulaire et rotatif à surface raclée. Le tableau 7 donne les résultats d'un essai effectué sur la tour B.

TABLEAU 7

Influence de la température du concentré sur les pertes par la cheminée

\begin{tabular}{c|c|c|c}
\hline $\begin{array}{c}\text { Température }\left({ }^{\circ} \mathrm{C}\right) \\
\text { du concentré }\end{array}$ & $\begin{array}{c}\text { Concentration } \\
\text { de poudre dans l'air } \\
\text { de séchage } \\
\mathrm{g} / \mathrm{m}^{3}\end{array}$ & $\begin{array}{c}\text { Concentration } \\
\text { de poudre dans l'air } \\
\text { évacué } \\
\mathrm{mg} / \mathrm{m}^{3}\end{array}$ & Perte p. 100 \\
\cline { 2 - 3 } 42 & 27,7 & 124 & 0,45 \\
55 & 29,0 & 125 & 0,43 \\
72 & 29,6 & 95 & 0,32 \\
\hline
\end{tabular}

Ces données montrent que malgré la pulvérisation plus fine de concentrés portés à une température plus élevée, l'influence exercée sur la perte de poudre a été favorable. Ceci est évidemment dû à la charge plus élevée du cyclone, qui se traduit dans les concentrations de poudre de l'air de séchage. Le chauffage du 
concentré résulte d'ailleurs facilement en poudres à particules de taible densité, à poids d'emballage et teneur en eau moins élevés, et à volume de vacuoles plus considérable.

3.2.4. Comparaison des pertes de poudre dans la fabrication de poudre de lait écrémé et de poudre de sérum

Il est généralement admis que le séchage du sérum entraîne des pertes de poudre notablement plus élevées que le séchage du lait écrémé ou entier. On impute ceci au fait que la poudre de sérum serait plus fine et (ou) plus légère.

Le tableau 8 reproduit les résultats d'un test effectué dans la tour $\mathrm{G}$, où du lait écrémé et du sérum ont été séchés successivement le même jour.

\section{$T A B L E A U 8$}

Données relatives à la fabrication et aux caractéristiques de la poudre de lait et de la poudre de sérum produits dans une même installation de séchage

\begin{tabular}{|c|c|c|}
\hline & $\begin{array}{l}\text { Poudre de lait } \\
\text { écrémé }\end{array}$ & $\begin{array}{l}\text { Poudre de } \\
\text { sérum }\end{array}$ \\
\hline \multicolumn{3}{|l|}{ Fabrication de la poudre : } \\
\hline - extrait sec du concentré & 50,0 & 53,0 \\
\hline - concentration de poudre dans l'air de séchage & 24,3 & 23,5 \\
\hline - concentration de poudre dans l'air évacué & 93 & 471 \\
\hline - perte de poudre & 0,4 & 2,0 \\
\hline \multicolumn{3}{|l|}{ Caractéristiques de la poudre: } \\
\hline — poids d'emballage : $\mathrm{g} / \mathrm{cm}^{3}$. & 0,74 & 0,72 \\
\hline - volume des vacuoles : $\mathrm{cm}^{3} / 100 \mathrm{~g}$ & 16,87 & 18,83 \\
\hline — densité des particules : $\mathrm{g} / \mathrm{cm}^{3}$ & 1,19 & 1,17 \\
\hline $\begin{array}{l}\text { - granulométrie (microscope) : } \\
\text { p. } 100 \text { entre } 0-16,5 \mu \\
\text { p. } 100 \text { entre } 16,5-33 \mu \\
\text { p. } 100 \text { entre } 33-66 \mu \\
\text { p. } 100 \text { entre } 66-99 \mu \\
\text { p. } 100 \text { de plus de } 99 \mu\end{array}$ & $\begin{array}{r}12,4 \\
44,8 \\
40,2 \\
2,4 \\
0,2\end{array}$ & $\begin{array}{r}19,9 \\
47,2 \\
28,9 \\
3,4 \\
0,6\end{array}$ \\
\hline
\end{tabular}


Ces résultats confirment de façon suffisamment nette que la perte en poudre de sérum a été considérablement plus élevée que la perte en poudre de lait écrémé. Les caractéristiques physiques mentionnées démontrent que la poudre de sérum avait des grains nettement plus fins que la poudre de lait, mais n'était qu'à peine plus légère, avec un volume de vacuoles seulement un peu plus grand.

\section{CONCLUSION}

De nombreux mesurages de la perte de poudre ont été effectués pendant une période de 2 ans dans différentes installations d'atomisation industrielle. Chaque mesurage avait une durée de $30 \mathrm{mn}$.

Le cyclone utilisé convenait fort bien pour cet usage et la méthode d'essai simplifiée, qui consistait en seulement un ou deux mesurages en deux points différents du flot de poudre, a été considérée comme suffisamment fiable. Les différences entre les résultats de mesurages répétés effectués en un même point ou en différents points ont été imputées à des variations des conditions d'exploitation de l'installation.

Les pertes de poudre relevées dans les différentes installations présentaient d'assez grandes divergences. Importants sous ce rapport n'étaient pas seulement la nature de l'installation et celle du produit séché, mais aussi le mode de séchage et les propriétés physiques correspondantes du produit. Les taux de pertes variaient entre 0,25 p. 100 et 1,35 p. 100 pour la poudre de lait écrémé et entre 1 p. 100 et 3,6 p. 100 pour la poudre de sérum. Des températures plus élevées de l'air de séchage et du concentré et, surtout, de plus fortes teneurs en extrait $\mathrm{sec}$ du concentré ont influé favorablement sur les pertes de poudre.

Le fait que les pertes de poudre sont nettement plus considérables dans la fabrication de poudre de sérum que dans celle de poudre de lait écrémé est principalement dû à la distribution granulométrique des particules de poudre.

Dans les conditions économiques actuelles, il est d'autant plus important de contrôler et d'optimiser la température de l'air de séchage, la température du concentré et, surtout, le degré de concentration du produit à atomiser, que l'influence de ces facteurs s'exerce dans le même sens, favorable ou défavorable, sur la perte de poudre et sur la consommation d'énergie. 


\section{Remerciements}

Les auteurs tiennent à exprimer à M.W. De Ville, ingénieur-technicien, leur gratitude pour la précieuse assistance technique qu'il leur a accordée.

\section{Ré s u m é}

La quantité de poudre, entraînée par l'air sortant des installations d'atomisation et dispersée dans l'environnement, représente une perte de rendement et pose des problèmes de pollution de l'air.

Un grand nombre de mesurages de la quantité de poudre ainsi perdue ont été effectués dans sept installations industrielles au moyen d'un équipement de mesure convenant pour l'emploi pratique.

Les résultats, exprimés en pourcentage de la quantité de poudre produite, ont varié, d'une installation à l'autre entre $0,25 \mathrm{p} .100$ et 1,35 p. 100 pour la poudre de lait écrémé et entre 1 p. 100 et 3,60 p. 100 pour la poudre de sérum. Les résultats relatifs à chaque intallation présentaient toutefois des variations importantes. En ce qui concerne les variables technologiques étudiées, il est apparu que l'augmentation des températures de l'air à l'entrée et du concentré et, surtout de plus hautes teneurs en extrait sec du concentré exercent une influence favorable.

\section{S u m m a r y}

The quantity of powder blown outside with the exhaust air of spray-drying plants both means a loss in efficiency and poses problems of air pollution.

The exhaust air of seven industrial plants was repeatedly measured for the loss in powder; this was achieved by means of a measuring instrument suitable for practical use.

The powder loss recorded in the different plants, expressed in powder quantity, ranged from 0.25 to $1.35 \mathrm{p} .100$ for skim milk powder and from 1 to $3.60 \mathrm{p} .100$ for whey powder. The loss recorded in individual plants was, however, also highly divergent.

Among the process variables investigated, mainly the dry matter content of the concentrate and, to a less extent, the temperature of the inlet air and the concentrate played an important part: favourable results were noted when these variables were increased. 


\section{Références bibliographiques}

Buma (T. J.) (1971). - Free fat in spray-dried milk. Neth. Milk Dairy J., 25, 123.

de Vilder (J.), MaRTens (R.) and NaudTs (M.) (1976). - Influence of process variables on some whole milk powder characteristics. Milchwissenschaft, $31,396$.

De Vilder (J.) and Martens (R.). - De invloed van de indampingsgraad, het homogenizeren en het verhitten van het koncentraat op de fysische eigenschappen van volle melkpoeder. (Ter perse).

Duiven (J.E.). (1967). - Ervaringen opgedaan met de Capillair Impinger voor de bepaling van het gehalte aan melkpoeder in de uitlaatlucht van verstuivingsinstallaties. Officieel Org. K. ned. Zuivelbond., 59, 1148.

Hafenmayer (F.) (1972). - Sprühtrocknung mit Entstaubung der Abluft. Deutsche Molk. Ztg., 93, 1608.

Hawksley (P. G. W.), Badzioch (S.) and Blackett (J. H.) (reprint 1971). - Measurement of solids in flue Gases. The British Coal Utilisation Research Association, Leatherhead, Surrey, England.

FIL/IDF 26 (1964). - Determination of the water content of dried milk. International dairy Federation, Brussels. 